Enfermagem Brasil 2018;17(4):326-35

\title{
ARTIGO ORIGINAL \\ Utilização do telessaúde na educação permanente pelos enfermeiros da Estratégia da Saúde da Família
}

\author{
Denilien Brown, Esp*, Luciane Bisognin Ceretta, D. Sc. ${ }^{* *}$, Maria Tereza Soratto, M.Sc. ${ }^{* \star *}$ \\ *Enfermeira, Especialista em Gestão da Atenção Básica de Saúde, Universidade do Extremo \\ Sul de Santa Catarina (UNESC), Criciúma/SC, ${ }^{* \star}$ Enfermeira, Mestre em Enfermagem UNESC, \\ ${ }^{* * *}$ Enfermeira, Mestre em Educação UNESC
}

Recebido em 11 de setembro de 2017; aceito em 8 de janeiro de 2018.

Endereço para correspondência: Maria Tereza Soratto, UNESC, Av. Universitária, 1105, Curso de Enfermagem, Bloco S, Bairro Universitário, 88806-000 Criciúma SC, E-mail: Denilien Brown: denilibrown@yahoo.com.br; Luciane Bisognin Ceretta: luk@unesc.net; Maria Tereza Soratto: guiga@unesc.net.

Artigo Baseado em Monografia de Pós-graduação Especialização em Gestão da Atenção Básica de Saúde

\section{Resumo}

Estudo com objetivo de identificar os desafios enfrentados pelas enfermeiras da Estratégia Saúde da Família para utilização do Programa Telessaúde. Pesquisa de abordagem qualitativa, descritiva, exploratória e de campo. Aplicou-se entrevista semiestruturada com seis enfermeiras da Estratégia Saúde da Família em um município do litoral norte do Rio Grande do Sul. A análise dos dados foi realizada a partir da análise de conteúdo, segundo Minayo. A principal dificuldade citada pelas enfermeiras para a utilização do Telessaúde foi relacionada à falta de tempo, a cultura dos profissionais que interfere na adesão para a utilização de recursos de educação à distância e a falta de capacitação para o uso do programa. O Telessaúde é primordialmente utilizado pelas enfermeiras para a organização de palestras, como material de apoio e para a capacitação da equipe, porém a adesão ao programa não ocorre de forma integral pela equipe multiprofissional da ESF. O Programa Telessaúde abre novas possibilidades de atenção à saúde, sendo necessário que a equipe multiprofissional se aproprie desta tecnologia de forma integral objetivando a qualificação da assistência com a utilização de recursos tecnológicos. Sugere-se capacitação das equipes, tornando o programa uma ferramenta de uso multiprofissional, diário e contínuo na sua totalidade.

Palavras-chave: enfermagem, educação em saúde, educação continuada, tecnologia da informação, estratégia saúde da família.

\footnotetext{
Abstract

Use of telehealth on permanent education by nurses of the Family Health Strategy

Study aiming to identify the challenges faced by nurses of the Family Health Strategy for using Telehealth Program. This is a qualitative, descriptive, exploratory approach and field research. A semi-structured interview was applied to six nurses of the Family Health Strategy in a city in the North coast of Rio Grande do Sul. Data analysis was carried out from the analysis of content, according to Minayo. The main difficulty cited by nurses for Telehealth utilization was related to lack of time, the culture of the professionals who interferes with the membership for the use of distance education resources and the lack of training for the use of the program. The Telehealth is primarily used by nurses to the Organization of lectures, as support material and to the training of the team, and adherence to the program occurs fully by the multidisciplinary team of the family health strategy. The Telehealth Program opens new possibilities for health care, being necessary for the multiprofessional team to take ownership of this technology fully in order to qualify the assistance with the use of technological resources. It was suggested training of teams, making the program a tool for multidisciplinary use, daily and continuous in its entirety. Key-words: Nursing, health education, education, continuing, information technology, Family Health Strategy.
} 


\section{Resumen \\ Uso de la telesalud en educación permanente por personal de enfermería de la Estrategia de Salud de la Familia}

Estudio con el objetivo de identificar los desafíos que enfrentan las enfermeras del Estrategia de Salud Familiar para el uso del programa de telesalud. Investigación de enfoque cualitativo, exploratorio, descriptivo y de campo. Se aplicó una entrevista semiestructurada a seis enfermeras de Estrategia de Salud Familiar en un municipio de la costa norte de Rio Grande do Sul. Análisis de datos se llevó a cabo a partir del análisis de contenido, segundo Minayo. La principal dificultad citada por las enfermeras para utilización de telesalud se relacionó con falta de tiempo, la cultura de los profesionales que interfiere con la calidad de miembro para el uso de recursos de educación a distancia y la falta de capacitación para el uso del programa. La telesalud es utilizada principalmente por las enfermeras para la organización de conferencias, como material de apoyo y la formación del equipo, y la adhesión al programa se produce completamente por el equipo multidisciplinario del Estrategia de Salud Familiar. El programa de telesalud abre nuevas posibilidades para el cuidado de la salud, siendo necesario para el equipo de multiprofesional a tomar posesión de esta tecnología completamente para recibir la asistencia con el uso de recursos tecnológicos. Se sugiere la formación de equipos, haciendo del programa una herramienta para uso multidisciplinario, diaria y continua en su totalidad.

Palabras-clave: Enfermería, educación en salud, educación continua, tecnología de la información, estrategia de salud familiar.

Introdução

A Educação Permanente (EP) em Saúde configura a Educação em Serviço, caracteriza-se como uma estratégia a fim de transformar as práticas de saúde. Ela é realizada a partir dos problemas encontrados na realidade de cada contexto regional e leva em consideração a experiência e o pré-conhecimento de cada profissional. Os processos de educação permanente deverão ser pautados nas necessidades daquelas populações adscritos, por isso é tão importante a problematização dos processos de trabalho e isso se garante com a educação permanente $[1,3]$.

Tendo o Sistema Único de Saúde (SUS) como o modelo vigente de saúde no Brasil, vários movimentos de fortalecimento deste sistema foram iniciados a fim de alcançar as diretrizes do SUS. Para tanto, a própria Constituição Federal de 1988, define que ao SUS compete ordenar a formação de recursos humanos na área de saúde [1,3]. Iniciar esta estratégia de Educação Permanente na Atenção Básica à Saúde (ABS) é condição fundamental para a consolidação de mudanças no modelo de atenção à saúde, pois é lá que o usuário terá seu primeiro contato com a saúde, é nesta esfera que cada vez mais este setor deverá ser fortalecido para ser resolutivo e eficiente.

A Política Nacional de Educação Permanente em Saúde (PNEPS) e a Política Nacional de Humanização aprovadas e instituídas no Brasil em 2003/2004 fomentaram a reorientação da formação dos profissionais em saúde. As Diretrizes Curriculares Nacionais propostas pelo Ministério da Educação em 2001, buscou a construção de um perfil de profissional que contemplasse às necessidades da população, o sistema de saúde vigente no país, o trabalho em equipe e a atenção integral à saúde [4].

Outra ação criada pelo Ministério da Saúde foi o Telessaúde Brasil Redes na Atenção Básica, componente do Programa de Requalificação das Unidades Básicas de Saúde (UBS), instituído pela Portaria o 2.554 de 28 de outubro de 2011. O objetivo principal desta ferramenta é potencializar a qualificação da Atenção Básica/Estratégia de Saúde da Família, estimulando o uso das Tecnologias de Informação e Comunicação (TICs) para atividades à distância relacionadas à saúde. O Telessaúde pode possibilitar a interação entre profissionais de saúde, bem como o acesso remoto a recursos de apoio educacional, clínicos e diagnósticos [5].

Telessaúde é uma ferramenta de articulação entre a atenção especializada e a atenção básica, pois estimula uma nova forma de comunicação entre esses pontos de atenção. A ampliação do diálogo entre os pontos de Atenção Especializada e Atenção Básica é essencial para aumentar a resolutividade da Atenção Básica e qualificar o acesso do usuário aos serviços especializados. O Telessaúde ainda é pouco explorado pelas equipes sendo uma grande ferramenta para o movimento de mudança proposta para qualificação do cuidado na Atenção Básica $(A B)$ e melhoria do acesso à Atenção Especializada (AE) no SUS [5]. 
A Educação Permanente em Saúde (EPS) é um dispositivo capaz de aproximar o cotidiano do profissional do SUS e as necessidades da população. Desenvolve a reflexão crítica dos profissionais no processo de trabalho ao ponto que compreendam a complexidade da realidade dinâmica que é o serviço saúde. Desenvolver ferramentas que agucem a EPS nas equipes multidisciplinares é uma estratégia essencial para a melhoria da assistência e para o crescimento profissional do trabalhador da saúde.

A saúde é um processo de trabalho que está sempre em transformação, o profissional deverá estar sempre em movimento de aprendizagem. O conhecimento se constrói a partir da problematização da realidade, da articulação com a teoria e a prática, do diálogo da equipe, da troca de saberes. A construção do saber é coletiva, crítica e desenvolvida no contexto social. $O$ programa Telessaúde Brasil Redes surge para fomentar a questão da EPS dentro das equipes da ESF. É uma ferramenta inovadora para o aprendizado e crescimento profissional.

Os avanços tecnológicos modificaram as formas de processamento, disseminação, armazenamento e recuperação de informações. Neste sentido o Telessaúde aparece como equalizadora de demandas que podem proporcionar novas formas de transmissão de conhecimentos e cuidados em saúde, auxiliando na melhoria da atenção à saúde e contribuindo para a diminuição dos índices de mortalidade e morbidade, gerando intervenções mais arrojadas e rápidas, evitando grandes deslocamentos para grandes centros urbanos e possibilitando o acesso aos cuidados da saúde em localidades remotas [6].

Percebe-se que muitos municípios adotaram este programa e estão cadastrados para a utilização da ferramenta, mas ainda é pouco utilizado na sua integralidade pela equipe multiprofissional. Atualmente ainda ocorrem nas equipes da ESF capacitações pontuais, reuniões para informes e não movimentos de EPS. As coordenações da ESF devem avaliar as temáticas a partir da necessidade e percepção de suas próprias equipes para a realização de capacitações e reuniões. O termo "educação continuada" também é fortemente confundido com EPS pela própria gestão e equipe multiprofissional. Considera-se que a equipe multiprofissional nem sempre utiliza esta ferramenta tecnológica em toda a sua capacidade, e o Programa Telessaúde pode dar subsídios a Educação Permanente em Saúde realizada na ESF.

Nesta perspectiva, este estudo teve por objetivo identificar de que forma o Telessaúde é utilizado na Educação Permanente pelos enfermeiros da Estratégia da Saúde da Família (ESF).

Material e métodos

Pesquisa de abordagem qualitativa, descritiva, exploratória e de campo. Aplicou-se entrevista semiestruturada com seis enfermeiras da Estratégia da Saúde da Família de um município do litoral norte do Rio Grande do Sul. Utilizaram-se como critérios de inclusão dos participantes da pesquisa: enfermeiros atuantes na ESF; aceitação para participar da Pesquisa segundo Resolução 510/2016[7] e assinatura do Termo de Consentimento Livre e Esclarecido (TCLE).

$\mathrm{Na}$ entrevista questionaram-se os profissionais sobre a capacitação recebida relacionada à utilização do Telessaúde; facilidades e dificuldades para a utilização do programa; como o Telessaúde é utilizado na Educação Permanente pelos enfermeiros da Estratégia da Saúde da Família (ESF); a adesão da equipe multiprofissional na utilização do Telessaúde; o Programa Telessaúde e a qualificação da assistência na ESF; além de sugestão sobre a temática.

A aplicação do instrumento de coleta de dados foi realizada por uma das enfermeiras pesquisadoras, ocorrendo no horário de trabalho, individualmente, com a duração de 30 a 40 minutos; em ambiente adequado para garantir a privacidade das informações e o bem-estar do profissional.

A análise e interpretação dos dados qualitativos foram organizadas a partir da análise de conteúdo com a categorização dos dados, através da ordenação, classificação e análise final dos dados pesquisados, de acordo com Minayo [8]. Na ordenação dos dados faz-se um mapeamento de todos os dados obtidos no trabalho de campo, com a transcrição de gravações, releitura de material, organização dos relatos. Em seguida na classificação dos dados realizase a leitura exaustiva e repetida dos textos, estabelecendo interrogações para identificar o que surge de relevante, estabelecendo as categorias específicas. E na análise final articulam-se os dados e os referenciais teóricos da pesquisa, respondendo às questões da pesquisa com base em seus objetivos [8]. 
Um dos procedimentos mais úteis para a investigação qualitativa é a formulação e organização dos dados em categorias [9]. Categoria refere-se a um conceito que abrange elementos ou aspectos com características comuns ou que se relacionam entre si, são estabelecidas para classificar os eventos. Categorizar é agrupar elementos, ideias ou expressões em torno de um conceito $[8,9]$.

De acordo com a Resolução 510/2016, os participantes devem ser esclarecidos sobre a natureza da pesquisa, seus objetivos, métodos, benefícios previstos, potenciais riscos e o incômodo que esta possa lhes acarretar, na medida de sua compreensão e respeitados em suas singularidades [7]. Na pesquisa utilizou-se um termo de consentimento livre e esclarecido, informando aos participantes da pesquisa os objetivos, métodos, direito de desistir da mesma e sigilo em relação à pesquisa. Para preservar o sigilo e o anonimato dos participantes da pesquisa, de acordo com as diretrizes e normas regulamentadoras da Resolução 510/2016[7], utilizou-se indicador alfanumérico (E de Enfermeiro - E1 a E6). A pesquisa foi aprovada pelo Comitê de Ética em Pesquisa da UNESC pelo Projeto no 1.343.003/2015.

Resultados e discussão

\section{Perfil dos enfermeiros atuantes na ESF}

Em relação ao perfil das enfermeiras, todas eram do sexo feminino, a idade variou de 29 a 34 anos, tendo como média 31 anos. O tempo de atuação como enfermeira de ESF variou de 1 a 7 anos; tendo como média 3,5 anos de trabalho. As especializações das enfermeiras foram em Saúde da Família (E2); Auditoria em Saúde (E4); Urgência e Emergência (E1, E3 e E5); Educação Permanente em Saúde (E6); Nefrologia e Trauma (E3).

\section{Capacitação dos profissionais relacionados à utilização do Telessaúde}

Em relação à capacitação dos profissionais sobre a utilização do Telessaúde, os enfermeiros E2; E3 e E6 não foram capacitados, o que pode interferir diretamente na utilização desta ferramenta.

\footnotetext{
E2 - "Não tive nenhuma capacitação para o uso do programa, apenas quem teve foi o médico da unidade".

E3 - "Não tive nenhum tipo de capacitação para a utilização desta ferramenta. A meu ver há certa carência em relação às capacitações realizadas entre as equipes para utilização do Telessaúde. Muitos profissionais não utilizam esta ferramenta, muitas vezes porque não receberam nenhuma instrução técnica de como funciona o serviço". E6 - "Não possuo capacitação".
}

Os enfermeiros E1; E4 e E5 receberam capacitação sobre Telessaúde, e o enfermeiro E4 ainda recebeu feedback da equipe técnica do programa.

\footnotetext{
E1 - "Tive uma capacitação em 2013. O resto da equipe não possui capacitação".

E4 - "Foi realizada capacitação para a equipe e após o Telessaúde entrou em contato várias vezes para saber como estávamos com o sistema e se já havia equipamentos tecnológicos para o uso".

E5 - "Tive uma capacitação em 2013, quando este programa foi lançado nas ESFs do nosso município".
}

A capacitação dos profissionais de saúde de APS/ESF é realizada pelos coordenadores e monitores de campo (equipe técnica do Telessaúde) e tem como objetivo desenvolver as competências e habilidades necessárias para a utilização dos recursos disponibilizados pelo programa [10].

Conforme o Manual Instrutivo do Telessaúde lançado pelo Ministério da Saúde em 2012, todos os municípios deverão receber, via secretaria ou coordenadoria estadual de saúde, um comunicado referente à necessidade de capacitação e onde será dada a possibilidade do município escalonar a saída dos profissionais em turnos e datas diferentes, conforme as opções do cronograma para aquela região, de modo a não afetar a dinâmica de trabalho das equipes de saúde. Profissionais não capacitados não poderão usar o projeto e terão que aguardar por outras fases de capacitação ou por capacitação à distância, via webconferência de acompanhamento. O manual sugere ainda priorizar os profissionais de nível superior na 
primeira fase, alocando os demais profissionais nas fases seguintes ou durante a visitação técnica [10].

\section{As principais facilidades para a utilização do programa}

Para os enfermeiros entrevistados, a principal facilidade para a utilização do Telessaúde está relacionada à conectividade e comunicação (E1; E2; E3), realização de encaminhamentos (E4; E5; E6), além da possibilidade de utilização do programa em capacitações, como material de apoio e palestras (E4).

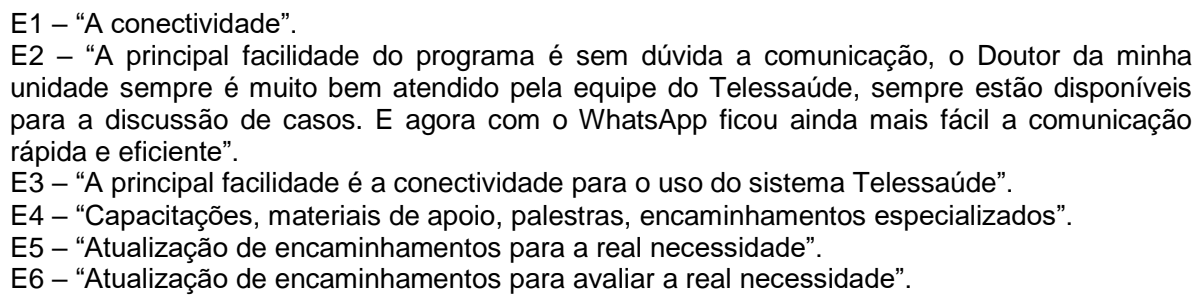

O Telessaúde é uma ferramenta de articulação entre a atenção especializada (AE) e a atenção básica $(A B)$, que provoca uma nova forma de comunicação entre esses pontos de atenção. A ampliação do diálogo entre os pontos de Atenção Especializada e Atenção Básica aumenta a resolutividade da Atenção Básica e qualifica o acesso do usuário aos serviços especializados. Esta ferramenta tem potencial para promover mudanças no processo de trabalho das equipes de Atenção Especializada, pois exige que a equipe desse ponto de atenção realize outras atividades. Entre elas, teleconsultoria, telediagnóstico, apoio matricial, reuniões, discussão de casos, apoio à regulação, desenvolvimento de protocolos e diretrizes e definição compartilhada de fluxos. Saindo do modelo tradicional (ambulatorial), realizando apenas consulta para usuários e estando isolado do restante da rede de serviços, esse ponto de atenção pode passar a se comprometer com o cuidado integral e em rede e não só com sua responsabilidade específica em cada caso, exercendo também funções que apoiam a clínica na atenção básica e a gestão das ações e serviços de saúde [5].

A teleconsultoria é um espaço de diálogo caracterizado pela comunicação eletrônica ou por telefone, no qual os profissionais podem tirar dúvidas e discutir casos; utilizadas como apoio diagnóstico por meio de exames que permitam a realização do telediagnóstico. Esses contatos quando feitos por meio eletrônico ou telefônico podem ser síncronos (no momento do cuidado) e assíncronos (em momentos fora da consulta) [5]. As tecnologias da informação aplicadas à educação potencializam a disseminação global do conhecimento, provocando o intercâmbio com o resto do mundo, conduzindo a individualização de seu acesso e aprendizado, através dos fluxos que determinam onde, quando, quem e como utilizá-los [1].

\section{As principais dificuldades para a utilização do Programa}

Por depender da mudança no processo de trabalho, suporte continuado (ex: financiamento, treinamento para profissionais de APS/ESF, disponibilidade de especialistas treinados e equipe técnica para manutenção), o sucesso na implantação destes serviços requer planejamento adequado e sua qualidade deve estar em permanente avaliação [10].

O tempo indisponível para a utilização do Telessaúde foi a principal dificuldade citada pelos enfermeiros $\mathrm{E} 1$; E2; E4; E5:

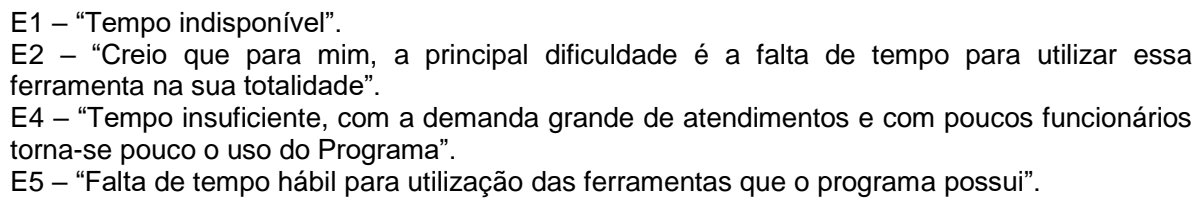

Para o enfermeiro E3 a cultura dos profissionais foi citada como principal dificuldade da utilização da ferramenta tecnológica: E3 - "As principais dificuldades estão relacionadas com a cultura de alguns profissionais de saúde no uso de tecnologias e das conectividades". 
A revolução tecnológica no último quarto do século $X X$ privilegiou os processos e a inovação contínua dos produtos, sendo a matéria-prima fundamental a informação e o conhecimento e operando sobre a produção de articulações, redes e fluxos entre as atividades e as organizações. Neste cenário, as organizações requerem o desenvolvimento da capacidade de flexibilidade e adaptabilidade das transformações, assim como da formação de alianças em áreas específicas de ação [1].

A falta de capacitação para o uso do Telessaúde foi considerado pelo enfermeiro E6 a principal dificuldade para a utilização do programa: E6 - "Falta de capacitação".

De maio a setembro de 2014, foi realizada uma pesquisa nacional de avaliação do programa Telessaúde, realizada pela Ouvidoria do Ministério da Saúde com o objetivo de verificar a implantação e utilização do Programa Telessaúde Brasil Redes. A pesquisa foi realizada por meio de inquérito telefônico operacionalizado pelos atendentes do Disque Saúde 136, para um público alvo de 119.108 profissionais de saúde de 19.906 Unidades Básicas de Saúde. Foram entrevistados 17.365 profissionais de saúde, destes 8.408 relataram conhecer o Telessaúde $(48,4 \%)$. Dentre os profissionais, os médicos e enfermeiros são os profissionais que mais conhecem o programa. Contudo, nos questionamentos sobre a utilização, verificou-se que dos profissionais que conhecem o programa apenas $40 \%$ o utilizam no seu dia a dia. É notório que o Programa sente a necessidade de métodos que ajudem a divulgar suas ações, como também capacitar um maior número de profissionais [11].

A tele-educação vem auxiliando neste aspecto através dos seus vários métodos de ensino à distância. Um deles vem ganhando destaque na área da saúde, que é o curso autoinstrucional. Este curso é um modelo de ensino à distância que vem se popularizando nos últimos anos. Ele se diferencia dos demais, pois garante autonomia e independência do aluno através de um material autoexplicativo, objetivo e simples, de forma que o aluno saiba conduzir o curso, permitindo que os alunos possam se matricular de forma independente e a qualquer momento, ficando livre para cursar o material disponível de acordo com a sua disponibilidade de tempo. O curso disponibiliza de forma acessível uma unidade com uma visão geral sobre o Programa Nacional Telessaúde Brasil Redes, e também possui outras unidades ensinando a utilizar o serviço de Teleconsultoria, formando Teleconsultores e Telerreguladores para atuarem nos mais diversos Núcleos pelo Brasil. Os trabalhadores da saúde podem acessar o site do ambiente virtual de aprendizagem, se cadastrarem de forma independente, e ter acesso aos materiais do curso. Como um dos objetivos do Programa é o de capacitar um maior número de trabalhadores da saúde, a criação de um curso autoinstrucional se torna uma importante estratégia para este fim [11].

O curso autoinstrucional em telessaúde permite que os trabalhadores de saúde adquiram maiores conhecimentos sobre o Programa, sejam capacitados para utilizarem o serviço de teleconsultoria, e possam atuar como teleconsultores e telerreguladores, através de um material didático e interativo que foi criado para facilitar o aprendizado dos alunos [11].

A utilização do telessaúde na educação permanente pelos enfermeiros da Estratégia da Saúde da Família (ESF)

Os serviços de saúde são impulsionados a buscar continuamente informação e conhecimento. A educação é uma temática constante dos debates da área da saúde, pois é o alicerce para a construção dos saberes. Com o aceleramento de inovações tecnológicas, várias mudanças ocorreram no âmbito social, cultural e econômico trazendo mudanças também nas formas de adquirir o conhecimento e a educação [12].

A Educação Permanente em Saúde (EPS) é um dispositivo essencial que deve ser ativado nas equipes da estratégia de saúde da família, pois possibilita o real desenvolvimento das diretrizes preconizadas no SUS. Neste enfoque questionaram-se as enfermeiras sobre a utilização do Telessaúde na Educação Permanente na Estratégia da Saúde da Família (ESF).

Os enfermeiros E3; E4 e E5 utilizam o Telessaúde como ferramenta para a Educação Permanente em Saúde através das webs palestras e dos materiais de apoio disponíveis no programa para a contínua capacitação e atualização da equipe:

E3 - "É utilizado principalmente através da web palestras".

E4 - "Através dos materiais de apoio".

E5 - "Quando possível é assistido as Web palestras e utilizado algumas informações para as capacitações da equipe". 
O aprendizado através de recursos tecnológicos, em particular na atualização profissional, atingiu grande desenvolvimento nas últimas décadas, permitindo a flexibilidade e acessibilidade ao conhecimento e à informação, superando problemas de distância e de acesso a bibliografias e potencializando a circulação de dados e o desenvolvimento de debates [1].

O Telessaúde não é utilizado como ferramenta na Educação Permanente, segundo o relato dos enfermeiros E1; E2 e E6:

E1 - "Não vem sendo usado para esse fim de educação permanente".

E2; E6 - "Não é utilizado desta maneira".

O Telessaúde tem potencial para facilitar o acesso aos serviços do sistema de saúde, aumentar a qualidade e contribuir para a formação profissional. No entanto, o seu potencial ainda não foi bem compreendido e a sua incorporação tem sido lenta $[5,13]$.

As novas tecnologias de informação e comunicação têm contribuído para o crescimento e a credibilidade da Educação a Distância (EAD). A EAD é uma importante ferramenta e estratégia para a educação permanente diante das novas tecnologias, sendo uma inovação pedagógica na educação. Este modelo pedagógico possibilita uma análise crítica da prática, permitindo refletir e problematizar as formas de prestação da assistência à saúde [14].

\section{A adesão da equipe multiprofissional na utilização do Telessaúde}

Segundo os enfermeiros a adesão do Telessaúde ocorre mais por parte dos médicos, mas os enfermeiros E5 e E6 utilizam-no conjuntamente com o médico da ESF:

\footnotetext{
E1 - "A médica da minha unidade é quem mais utiliza esta ferramenta para a discussão de casos de encaminhamentos para a especialidade".

E2 - "É utilizado pelo médico da minha unidade, eu particularmente nunca utilizo".

E5 - "Eu e o médico da minha unidade utilizamos para reavaliar encaminhamentos".

E6 - "Apenas eu e a médica da minha unidade utilizamos para avaliar a real necessidade de encaminhar um paciente para a especialidade".
}

multiprofissional:

O enfermeiro E3 e E4 ressaltaram a pouca adesão ao Telessaúde pela equipe

E3 - "Em minha opinião, a adesão da equipe multiprofissional na utilização do Telessaúde ainda é pouca, principalmente da parte médica, penso que muitos serviços oferecidos pelo serviço não são utilizados".

E4 - "Pouca adesão, devido ao tempo como citei anteriormente".

É importante que as equipes da ESF junto com a gestão municipal tenha o entendimento do programa e seus benefícios para a qualidade da atenção, tornando possível uma maior adesão do programa na sua integridade. Os resultados esperados com a adesão do telessaúde será a melhoria da qualidade do atendimento na Atenção Básica no Sistema Único de Saúde (SUS), com resultados positivos na resolubilidade do nível primário de atenção; expressiva redução de custos e do tempo de deslocamentos; fixação dos profissionais de saúde nos locais de difícil acesso; melhor agilidade no atendimento prestado; otimização dos recursos dentro do sistema como um todo, beneficiando, dessa forma, aproximadamente 10 milhões de usuários do SUS [10].

\section{O programa telessaúde e a qualificação da assistência na ESF}

O programa Telessaúde melhora a qualidade da assistência na ESF, com discussão dos casos clínicos, otimização do tempo entre a consulta médica, o diagnóstico, tratamento, análise e classificação adequada para o encaminhamento do paciente para especialidade.

E1 - "Melhora na questão da real indicação de encaminhamentos para a especialidade. São discutidos os casos e analisado a melhor indicação de tratamento e ou encaminhamento".

E2 - "Melhora a qualidade da assistência, pois o médico pode tirar dúvidas em relação à conduta necessária para cada paciente, discutindo casos e vendo a real necessidade dos encaminhamentos para a especialidade". 
E3 - "Qualifica o trabalho da equipe e o serviço prestado à população. Otimiza o tempo entre a consulta do paciente e o fechamento do diagnóstico médico".

E4 - "Facilita os encaminhamentos aos especialistas através da discussão de casos clínicos com os médicos".

E5 - "É importante para classificar encaminhamentos, sendo desnecessário o gasto com aqueles que não precisam mais".

E6 - "O programa é bom para avaliar encaminhamentos, são discutidos os casos clínicos para classificar a necessidade do paciente".

O emprego da EAD no Programa Nacional de Telessaúde tem um papel fundamental no atendimento em saúde, a partir do momento que proporciona ao profissional um novo conceito de educação e assistência, garantindo, assim, o intercâmbio de conhecimentos entre as instituições de ensino e os trabalhadores. O programa aumenta a resolutividade do serviço na atenção básica, reduzindo os encaminhamentos para os grandes centros urbanos, contribuindo assim para uma maior agilidade no atendimento e a melhoria da qualidade da assistência prestada na atenção básica no Sistema Único de Saúde [14].

Sugestão sobre a pesquisa: Utilização do Telessaúde na educação permanente pelos enfermeiros da Estratégia da Saúde da Família

As sugestões sobre a pesquisa foram relacionadas à busca de solução para efetivamente utilizar o programa em toda a sua capacidade, com objetivo de qualificar a assistência à saúde da população, sendo importante que toda a equipe multiprofissional utilize o Telessaúde, a partir de capacitação:

\footnotetext{
E1 - "Sugiro que através deste estudo seja criado soluções para uma melhor utilização do programa no seu todo".

E2 - "Sugiro que com esta pesquisa, esta ferramenta possa ser mais bem utilizada, não só pelo médico e sim por toda a equipe multidisciplinar".

E3 - "Sugiro que seja difundido ainda mais informações sobre o uso e a resolutividade que o Telessaúde tem para oferecer tanto para os profissionais quanto para a população".

E5 - "Sugiro que através desta pesquisa, seja utilizado mais o programa, para aperfeiçoar o atendimento das ESF's".

E6 - "Sugiro que através desta pesquisa, sejam implantados novos treinamentos para as equipes de ESF, para que assim possamos melhorar ainda mais a assistência à população".

O enfermeiro E4 não ofereceu sugestões: E4 - "Pesquisa bem elaborada, nada a sugerir".
}

Uma estratégia para a divulgação do programa e da própria atividade é a capacitação através da tele-educação. O tele-educação proporciona atividades educacionais ministrados à distância por meios de tecnologias da informação e comunicação. É uma atividade educacional que utiliza as ferramentas tecnológicas como meio para apoiar a formação de trabalhadores do SUS, de acordo com a Política Nacional de Educação Permanente em Saúde. Por exemplo, são ofertados cursos, módulos educacionais, web aulas/palestras em modalidade à distância [5].

Poucos estudos de boa qualidade metodológica avaliaram o impacto destas tecnologias nos desfechos clínicos, mas evidências têm demonstrado boa acurácia na maioria dos exames, redução do número de encaminhamentos, aumento da satisfação dos pacientes e redução dos custos. Por depender da mudança no processo de trabalho, suporte continuado (ex: financiamento, treinamento para profissionais de APS/ESF, disponibilidade de especialistas treinados e equipe técnica para manutenção), o sucesso na implantação destes serviços requer planejamento adequado e sua qualidade deve estar em permanente avaliação. É importante salientar que a criação de serviços de tele-diagnósticos não terá efeito se o tratamento não for disponibilizado para o paciente. Portanto, projetos nesta área devem ser pensados no contexto de uma rede de cuidado e não em substituição a ela [10].

Observa-se ainda insuficiente a produção científica voltada para inserção dos saberes e práticas de Telessaúde na esfera do SUS com o enfoque da Saúde Coletiva, sendo uma das lacunas a serem superadas. A literatura produzida a partir das experiências de Telessaúde no Brasil, em sua grande maioria, vincula-se ao pensamento da Clínica. Esta constatação fica mais evidente ao se buscar produção científica dedicada ao tema da avaliação de iniciativas de Telessaúde [15]. 
A principal facilidade citada pelas enfermeiras entrevistadas para a utilização do Telessaúde está relacionada à conectividade e comunicação, realização de encaminhamentos e a possibilidade de utilização do programa em capacitações, como material de apoio e palestras. Já a principal dificuldade citada pelas enfermeiras foi relacionada à falta de tempo disponível para a utilização do Telessaúde, a cultura dos profissionais que interfere na adesão para a utilização de recursos de educação à distância e a falta de capacitação para o uso do programa. O Telessaúde é primordialmente utilizado pelas enfermeiras para a organização de palestras, como material de apoio e para a capacitação da equipe, porém a adesão ao programa não ocorre de forma integral pela equipe multiprofissional das ESFs.

$\mathrm{Na}$ percepção das enfermeiras, o programa Telessaúde proporciona melhoria na qualidade da assistência na ESF, sendo necessário que a equipe utilize o programa em toda a sua capacidade. Desta forma sugere-se a sensibilização e capacitação das equipes, tornando o programa uma ferramenta de uso multiprofissional, diário e contínuo na sua totalidade. Como limitação do estudo considera-se o número limitado de profissionais participantes da pesquisa.

O Programa Telessaúde pode dar subsídios a Educação Permanente em Saúde realizada na ESF sendo considerada uma ferramenta de articulação entre a atenção especializada e a atenção básica. Considera-se que o Programa Telessaúde abre novas possibilidades de atenção a saúde, sendo necessário que a equipe multiprofissional se aproprie desta tecnologia de forma integral objetivando a qualificação da assistência com a utilização de recursos tecnológicos.

\section{Referências}

1. Brasil. Ministério da Saúde. Secretaria de Gestão do Trabalho e da Educação na Saúde. Departamento de Gestão da Educação em Saúde. Política Nacional de Educação Permanente em Saúde. Brasília: MS; 2009. 64 p.

2. Macêdo NB, Albuquerque PC, Medeiros KR. O desafio da implementação da educação permanente na gestão da educação na saúde. Trabalho, Educação e Saúde 2014;12(2):379-401.

3. Brasil. Ministério da Saúde. Secretaria-Executiva. Subsecretaria de Assuntos Administrativos. Educação Permanente em Saúde: um movimento instituinte de novas práticas no Ministério da Saúde: Agenda 2014. Brasília: MS; 2014. 120 p

4. Haddad AE, Brenellib SL, Passarella TM, Ribeiro TCV. Política Nacional de Educação na Saúde. Rev Baiana Saúde Pública 2008;32(1):98-114.

5. Brasil. Ministério da Saúde. Programa Nacional Telessaúde Brasil Redes: Custeio dos Núcleos de Telessaúde. Manual Instrutivo. Brasília: Ministério da Saúde; 2015. 32 p.

6. Assis EA. Processos Comunicacionais e Informacionais na Telessaúde: interações entre o ambiente de especialistas e a Atenção Básica no Sistema Único de Saúde SUS [Dissertação]. Rio de Janeiro: Instituto de Comunicação e Informação Científica e Tecnológica em Saúde; 2013. 126 p.

7. Brasil. Conselho Nacional de Saúde. Diretrizes e normas regulamentadoras de pesquisa envolvendo seres humanos. Resolução no 510/2016. Brasília: Conselho Nacional de Saúde; 2016.

8. Minayo MCS. Pesquisa social: teoria, método e criatividade. 28 ed. Rio Janeiro: Vozes; 2009. $108 \mathrm{p}$.

9. Leopardi MT. Metodologia da Pesquisa na Saúde. Florianópolis: UFSC; 2002. 294 p.

10. Brasil. Ministério da Saúde. Universidade Federal do Rio Grande do Sul. Manual de Telessaúde para Atenção Básica. Atenção Primária à Saúde. Brasília: MS; 2012. 123 p.

11. Figueiredo AM, Guedes TAL, Matos TM, Valentim RAM, Araujo BG, Guerra Neto CLB. Curso Autoinstrucional em Telessaúde: Uma visão geral. Rev Bras Inovação Tecnológica em Saúde 2015;5(4):43-50.

12. Carneiro VF, Brant LC. Telessaúde: dispositivo de educação permanente em saúde no âmbito da gestão de serviços. Rev Eletrônica Gestão \& Saúde 2013;4(2):494-16.

13. Santos AF, D'Agostino M, Bouskela MS, Fernandéz A, Messina LA, Alves HJ. Uma visão panorâmica das ações de telessaúde na América Latina. Rev Panam Salud Publica 2014;35(5-6):465-70. 
14. Godoy SCB, Guimarães EMP, Assis DSS. Avaliação da capacitação dos enfermeiros em unidades básicas de saúde por meio da telenfermagem. Esc Anna Nery 2014;18(1):148-55.

15. Silva AB. Política pública, educação, tecnologia e saúde articuladas: como a Telessaúde pode contribuir para fortalecer o SUS? [Tese]. Rio de Janeiro: Escola Nacional de Saúde Pública; 2013. 153 p. 